\title{
Cultural characteristics and cordycepin production of some Cordyceps militaris strains under artificial cultivation conditions
}

\author{
Luyen Thi Nguyen ${ }^{1}$, Ve Van Le ${ }^{2}$, Bich Thuy Thi NGuyen ${ }^{1}$, Nghien Xuan Ngo ${ }^{1}$, \\ HUYen Trang Thi NGUYen ${ }^{1}$, QUAN Dinh NGUYeN ${ }^{3}$, SiKandar MUlla ${ }^{4 *}$ \\ ${ }^{1}$ Vietnam National University of Agriculture, Hanoi, Vietnam \\ ${ }^{2}$ University of Science and Technology, Seoul, South Korea \\ ${ }^{3}$ National Institutes of Medicinal Materials, Hanoi, Vietnam \\ ${ }^{4}$ REVA University, Bangalore, India
}

\begin{abstract}
Cordyceps militaris, a precious medical mushroom, has attracted wide attention in industrial fields. Currently, the degeneration phenomenon of $C$. militaris commercial strains is amongst the major challenges for cultivation at the industrial scale. The screening for superior strains with high yield and medicinal value is considered a realistic approach to overcome degeneration problems. In the present study, the mycelial growth, primordia formation, yield performance, and cordycepin content of five strains (DT1, DT2, DT3, DT4, and DT5) under artificial cultivation conditions were investigated. All strains showed mycelial growth on SDAY and liquid medium. The strains were successfully cultivated in brown rice medium and required 18 (strain DT3) to 25 days (strain DT5) to form primordia. Additionally, morphological characteristics of fruiting bodies varied among the strains. Strains DT4 and DT3 exhibited the highest fruiting body length with $74.23 \pm 5.13 \mathrm{~mm}$ and $72.63 \pm 2.62 \mathrm{~mm}$, respectively whereas the highest diameter was recorded for strains DT1 $(4.05 \pm 0.18 \mathrm{~mm})$ and DT2 $(3.63 \pm 0.12 \mathrm{~mm})$. Of note, among the investigated strains, strain DT3 exhibited the highest biological efficiency $(8.95 \pm 0.07 \%)$ and cordycepin content $(1.68 \mathrm{mg} / \mathrm{g})$. Therefore, strain DT3 could be selected as a potential strain for commercial cultivation.
\end{abstract}

Key words: Cordyceps militaris, mycelial, fruiting body, cordycepin

\section{Introduction}

As one of the most precious medicinal fungi, Cordyceps militaris belonging to the genus Cordyceps, family Cordycipitaceae, and class Ascomycetes has received considerable attention in several fields, including medicinal herbs, pharmaceutical products, tonic foods, and biocontrol agents against pests (Akata et al., 2016; Cui et al., 2015; Chiu et al., 2016; Zhang et al., 2019; Das et al., 2010; Yang et al., 2014). The fruiting body of C. militaris contains several bioactive metabolites such as cordycepin, adenosine, inosine, mannitol, sterols, polysaccharides, and pentostatin (Cui et al., 2015). Earlier studies reported positive health effects of these compounds to humans in the treatment of cancer, inflammatory diseases, and diabetes and for immunity improvement (Sun et al., 2018; Yin et al., 2017; Zhao et al., 2014; Yang et al., 2014). Among the bioactive metabolites, cordycepin - a nucleoside analog of adenosine - is a chemical marker used to distinguish genus Cordyceps from other fungi (Chamyuang et al., 2019).

Owing to strict growth requirements, $C$. militaris is scarce in nature (Yin et al., 2017). Thus, in order to improve the equality between the supply and demand chain, especially in Asian countries, artificial cultivation of Cordyceps militaris has been found to be a useful method (Yin et al., 2017). Unlike other species in genus

\footnotetext{
*Corresponding author: REVA University, Bangalore, India; e-mail: sikandar.mulla@gmail.com
} 
Cordyceps, C. militaris can grow easily and form fruiting bodies on artificial media, and therefore, it is considered as the only species of this genus that could be cultivated for cordycepin production at the industrial scale (Zhang et al., 2019; Lin et al., 2017; Sun et al., 2018; Chamyuang et al., 2019). As described previously by Chiang et al. (2017), the cultivation of $C$. militaris could be classified into three periods based on growth characteristics (mycelium colonization, primordia formation, and fruiting body development stage). To improve biological efficiency, several parameters such as light conditions, temperature, humidity, $\mathrm{pH}$, nutrition source, and minerals should be optimized (Chamyuang et al., 2019). To cultivate $C$. militaris in a large scale, cereal grains were widely used as the primary carbon source (Lin et al., 2017). Rice, cottonseed shells, wheat grain, corn grain, millet, sorghum, and corn cob particles have been used as main substrates and nutrient sources for the production of fruiting bodies and bioactive compounds by $C$. militaris (Lin et al., 2017; Shrestha et al., 2012b). Several studies have revealed that during the cultivation of $C$. militaris, light is the key environmental factor in determining pigment formation, stroma production, and cordycepin and carotenoid formation (Chiang et al., 2017; Yang et al., 2016; Shrestha et al., 2012b; Shrestha et al., 2006). The optimal light intensity for fruiting body induction was found to be 500 to $1000 \mathrm{~lx}$ (Hong et al., 2010). The suitable light condition for the growth of fruiting body is the light/dark cycle of $12: 12 \mathrm{~h}$ at $25^{\circ} \mathrm{C}$ (Chen et al., 2011). Similar to light conditions, temperature is known to be one of the most significant physical factors that noticeably affect stroma production by $C . \mathrm{mi}$ litaris (Shrestha et al., 2012b). The optimum temperature for mycelial growth and stroma development of C. militaris was determined to be $20-25^{\circ} \mathrm{C}$ and $18-22^{\circ} \mathrm{C}$, respectively (Lee et al., 2010). The maintenance of high humidity of $70-90 \%$ has been recommended for the cultivation of $C$. militaris (Shrestha et al., $2012 \mathrm{~b}$ ). The optimal medium (yeast extract $10.33 \mathrm{~g} / \mathrm{l}$, sucrose $\left.27.24 \mathrm{~g} / 1, \mathrm{KH}_{2} \mathrm{PO}_{4} 5.60 \mathrm{~g} / \mathrm{l}\right)$ for the mycelial growth of $C$. militaris was a submerged culture obtained by response surface methodology (Yang et al., 2014).

Although $C$. militaris is known as a cultivable medicinal mushroom, the industry faces several huge challenges such as the high frequency of strain degeneration (Shrestha et al., 2012b; Sun et al., 2018; Yin et al., 2017). Such degenerated strains of $C$. militaris exhibit typical characteristics such as slow mycelium growth, reduced mycelial density, loss of the ability to form primordia, irregular shapes and sizes of fruiting bodies, excessive aerial hyphae, and poor development of stroma and fruiting bodies (Shrestha et al., 2012b; Sun et al., 2018; Wang et al., 2017). As reported by Yin et al. (2017), sub-culture is a major driving factor of the degeneration phenomenon of $C$. militaris. As observed, C. militaris started to degenerate at the third subcultured generation and therefore showed incomplete fruiting body development at the next generation. The mechanism of degeneration of $C$. militaris was directly related to genes responsible for toxin biosynthesis, DNA methylation, energy metabolism, and chromosome remodeling (Yin et al., 2017). To address the degeneration problem, the search for superior strains with high yield and medicinal value plays a crucial role in the cultivation of $C$. militaris at the industrial scale. In an attempt to look for potential strains, five strains -DT1, DT2, DT3, DT4 andDT5 were collected in Tam Dao mountain $\left(21^{\circ} 31^{\prime} 0^{\prime \prime} \mathrm{N}\right.$ $105^{\circ} 33^{\prime} 0^{\prime \prime} \mathrm{E}$ ), Vietnam and deposited into Mushroom Research and Development Center, Vietnam National University of Agriculture. Taken together, the present study investigated 1) cultural characteristics of five $C$. militaris strains grown on solid-state and liquid media; 2 ) the potential of cultivation of $C$. militaris strains; and 3 ) the ability of $C$. militaris strains to produce cordycepin.

\section{Materials and methods}

\section{Fungal strains}

C. militaris strains DT1, DT2, DT3, DT4, and DT5 used in this study were obtained from Mushroom Research and Development Center, Vietnam. Pure mycelial cultures were isolated from the fresh fruiting bodies and maintained on PGA (potato, glucose, and agar) slants according to the procedure of Lin et al. (2017).

\section{Culture conditions}

To characterize the mycelium grown on solid-state medium, C. militaris strains were cultivated on SDAY medium ( $20 \mathrm{~g} / 1$ dextrose, $5 \mathrm{~g} / \mathrm{l}$ yeast extract, $5 \mathrm{~g} / \mathrm{l}$ peptone, and $15 \mathrm{~g} / \mathrm{l}$ agar) at $25^{\circ} \mathrm{C}$ in dark. The diameter of the mycelium extension $(\mathrm{mm})$ was measured at 5 to 25 days (5-day interval). The morphological characteristics of mycelia such as texture, density, and color were assessed by visual observations. 
To characterize the mycelium grown in submerged cultures, 10 pieces (approximately $0.5 \times 0.5 \mathrm{~cm}$ ) of overgrown agar plugs were excised, transferred into $100 \mathrm{ml}$ sterile water, and homogenized. Precultures were grown in a $500 \mathrm{ml}$ flask containing $250 \mathrm{ml}$ of the medium $(20 \mathrm{~g} / 1$ glucose, $1 \mathrm{~g} / \mathrm{l}$ peptone, $1 \mathrm{~g} / 1$ yeast extract, $1 \mathrm{~g} / 1$ $\mathrm{MgSO}_{4} \cdot 7 \mathrm{H}_{2} \mathrm{O}$, and $2 \mathrm{~g} / 1 \mathrm{KH}_{2} \mathrm{PO}_{4}$ ) and kept on a rotary shaker incubator $(120 \mathrm{rpm})$ at $22^{\circ} \mathrm{C}$. The density, size, and morphology of pellets were recorded for 1- 25 days. The dry weight of mycelium was determined following the protocol reported by Park et al. (2001). The mycelial pellets were washed with distilled water and dried overnight at $70^{\circ} \mathrm{C}$ until a constant weight was achieved.

\section{Substrate preparation and cultivation}

C. militaris strains were cultivated in a brown rice medium ( $50 \mathrm{~g}$ of brown rice supplemented with $10 \mathrm{~g}$ of silkworm pupae powder) according to the method of Shrestha et al. (2012a). The liquid spawn was inoculated in the medium and incubated at $22^{\circ} \mathrm{C}$ with relative humidity of $60-70 \%$ in dark at the spawn running stage after 15 days of incubation. After complete colonization of the medium, the strains were grown at $20^{\circ} \mathrm{C}$ under $85-90 \%$ humidity with a dark/light cycle of $12: 12 \mathrm{~h}$ for fruiting body formation and development.

Mycelial growth, period of substrate colonization, period of primordia formation, number of stromata, and length of stroma were measured as described previously by Ngo et al. (2019). The mycelial growth rate was calculated using the formula:

$$
\mathrm{V}=\mathrm{D} / \mathrm{T},
$$

where $V$ is the mycelial growth rate ( $\mathrm{mm} /$ day), $D$ is the diameter growth $(\mathrm{mm})$, and $T$ is the incubation time (days).

The period of substrate colonization (days) was defined as the time required for the mycelium to completely colonize the full substrate. The period of primordia formation (days) was defined as the time required for the appearance of primordia after inoculation.

The primordia ratio was defined as the percentage of bottle exhibiting primordium. The average biological efficiency (BE) was determined according to the calculations by Nguyen et al. (2019):

$$
\mathrm{BE}(\%)=\frac{\text { Dry weight of mushrooms }}{\text { Dry weight of the substrate }} \times 100
$$

\section{Quantification of cordycepin content}

To evaluate the medicinal value of five strains, the cordycepin content was quantified by HPLC. Powder of fruiting bodies $(1 \mathrm{~g})$ was precisely weighed, added to $10 \mathrm{ml}$ of $80 \%$ ethanol $(\mathrm{v} / \mathrm{v})$ in a $50 \mathrm{ml}$ corning polypropylene centrifuge tube, and sonicated for 30 minutes using an ultrasonic machine. The extracted solution was filtered using a $0.22 \mu \mathrm{m}$ cellulose acetate membrane filter (Thermo Fisher Scientific). The standard for cordycepin was obtained from Sigma-Aldrich.

HPLC was performed on a Zorbax Eclipse XDB18 column $(250 \times 4.6 \mathrm{~mm} ; 5 \mu \mathrm{m})$ with a flow rate of $0.8 \mathrm{ml} / \mathrm{min}$ and the injection amount of $20 \mu \mathrm{l}$. The mobile phase consisted of acetonitrile:water $(8: 92, \mathrm{vol} / \mathrm{vol})$. The detection wavelength was set at $260 \mathrm{~nm}$.

\section{Statistical methods}

Statistical evaluation was performed using GraphPad Prism, version 7.0 (GraphPad Software, Inc., San Diego, CA, USA). Each treatment was replicated three times. Significant differences were indicated with letters and assessed using one-way ANOVA followed by Tukey's multiple comparison test at $P<0.05$.

\section{Results}

\section{Mycelial growth of C. militaris strains on SDAY medium}

As shown in Figure 1A and Figure 1B, the results indicate that all investigated strains exhibited the ability to grow on SDAY medium. In terms of texture, the five strains were similar to each other and showed only one type of texture (floccose) (Fig. 1A). The mycelial density of strains on the $25^{\text {th }}$ day was found to be compact. Statistically significant differences $(P<0.05)$ were observed in the growth rate among the strains. The mycelium colony diameter of the five strains ranged from 78.07 $\pm 6.57 \mathrm{~mm}$ (DT5) to $90.00 \mathrm{~mm}$ (DT4) on the $25^{\text {th }}$ day. Remarkably, compared to other strains, strains DT3 $(90.00 \mathrm{~mm})$ and DT4 $(90.00 \mathrm{~mm})$ showed a significantly higher growth rate on 15,20 , and 25 days of incubation (Fig. 1B). The colony color characteristics of all strains were observed to be white when grown in dark but yellow when exposed to white light.

\section{Mycelial growth of $C$. militaris strains in submerged culture}

Among the five strains used for this study, strains DT3 and DT4 adapted better to submerged culture con- 
A
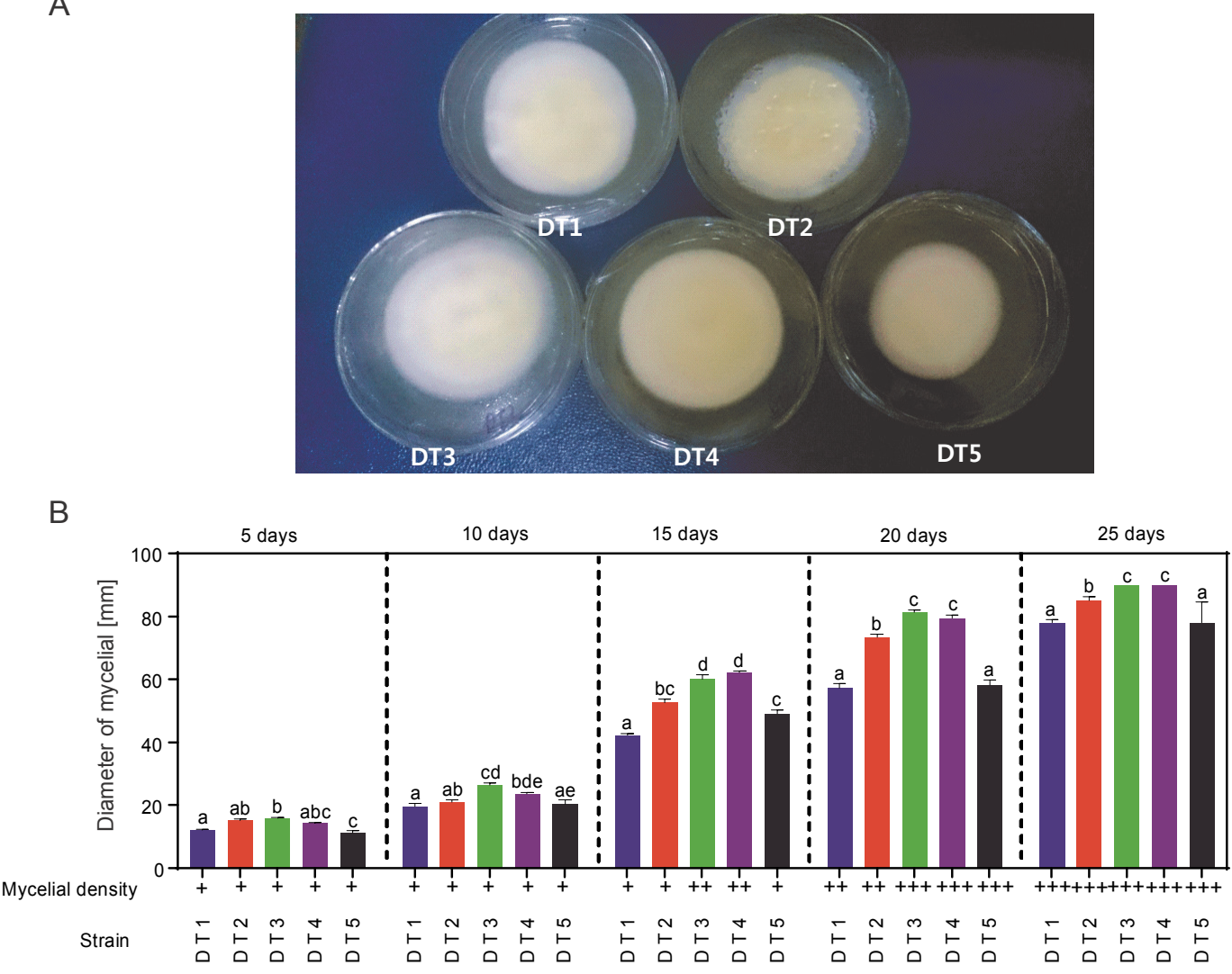

Fig. 1. A) Mycelial characteristics and B) growth of Cordyceps militaris strains on SDAY medium; bars in the same time period with different letters show significant differences at $P<0.05$

ditions than other strains. Under submerged culture conditions, the highest biomass production was obtained for DT4 and DT3 at 6 days after inoculation with 11.08 $\pm 0.65 \mathrm{~g} / \mathrm{l}$ and $11.04 \pm 0.18 \mathrm{~g} / \mathrm{l}$, respectively (Fig. 2C). Remarkably, both filamentous and pellet were identified as growth forms of all strains during cultivation in submerged conditions (Fig. 2A and Fig. 2B). The pellet morphology of the five strains was found to be hairy, fluffy, and circular in shape (Fig. 2B). The pellet diameter and density of all strains started to increase gradually after 48 hours from inoculation. However, a remarkable difference in pellet size was observed. Compared to other strains, the pellet size of strain DT5 $(1.11 \mathrm{~mm} /$ pellet $)$ was smaller after 6 days from inoculation.

\section{Cultivation characteristics of C. militaris strains}

As the first step to evaluate the cultivation potential of the five strains used in this study, their ability to produce primordia was analyzed. As shown in Figure 4, Figure 5, and Figure 6, all the five strains could form and develop primordia to the maturity stage. All the investigated strains required 18 days (DT3) to 25 days
(DT5) to form primordia (Fig. 3). The highest length of the fruiting body was found for strains DT4 and DT3 with $74.23 \pm 5.13 \mathrm{~mm}$ and $72.63 \pm 2.62 \mathrm{~mm}$, respectively (Fig. 5A). The highest diameter was observed for strains DT1 and DT2 $(4.05 \pm 0.18 \mathrm{~mm}$ and $3.63 \pm 0.12$, respectively) (Fig. 5B). As shown in Figure 5C, the number of fruiting bodies varied significantly among the strains and ranged from $8.53 \pm 0.16$ (DT1) to $120.07 \pm 2.46$ (DT3). The morphological characteristics of fruiting bodies of the investigated strains is shown in Figure 6A. Compared to other strains, the primordia ratio of strain DT1 showed a lower percentage of $68 \pm 6 \%$. The BE of the investigated strains varied significantly. Notably, the highest BE of $8.95 \pm 0.07 \%$ was observed for strain DT3 (Fig. 6B). Therefore, strain DT3 was found to be superior over other strains in terms of $\mathrm{BE}$ and should be used for commercial cultivation.

\section{Cordycepin content of $C$. militaris strains}

A calibration curve of cordycepin was constructed by plotting peak areas of the standard sample measured from $12.7 \mu \mathrm{g} / \mathrm{ml}$ to $203 \mu \mathrm{g} / \mathrm{ml}$. Cordycepin isolated from 
A
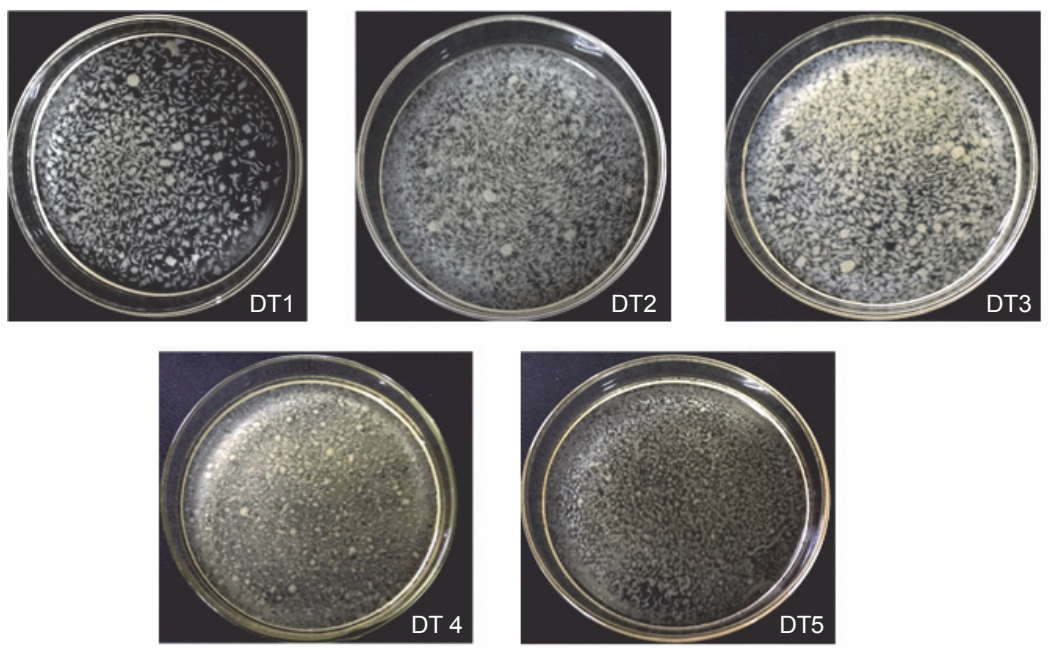

B
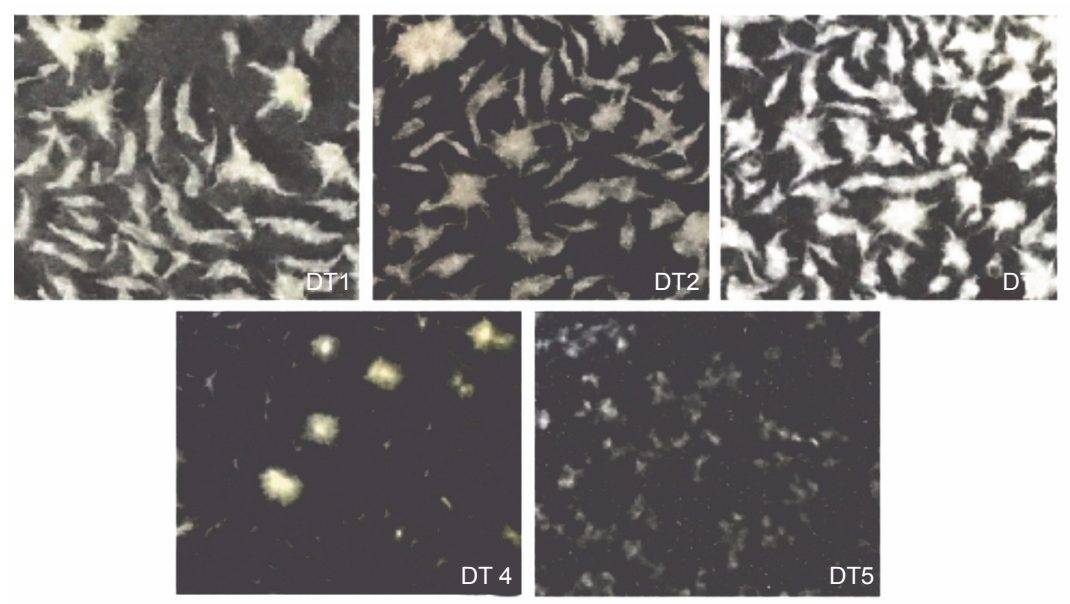

C

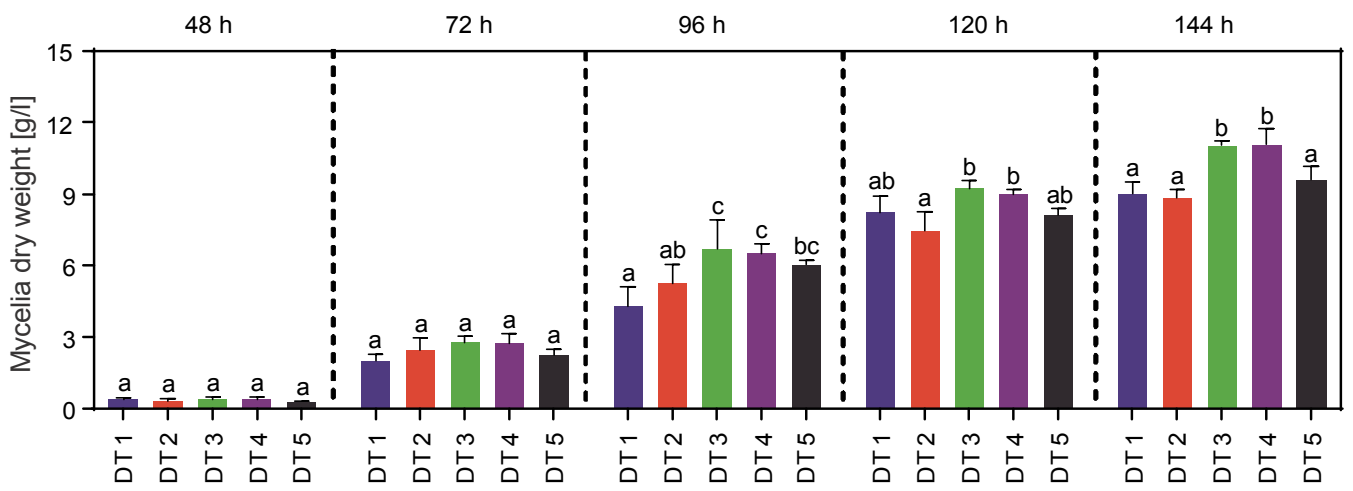

Fig. 2. A) Pellet density, B) morphology, and C) mycelia dry weight of Cordyceps militaris cultivated in submerged culture; bars in the same time period with different letters show significant differences at $P<0.05$

five strains was detected based on the retention time of samples and a standard (Fig. 7A). All strains exhibited the ability to produce cordycepin. However, in a detailed comparison, strain DT3 was found to produce cordycepin with the highest concentration $(1.68 \mathrm{mg} / \mathrm{g})$. In contrast, the lowest cordycepin content was observed for strain DT5 (0.24 mg/g) (Fig. 7B). 


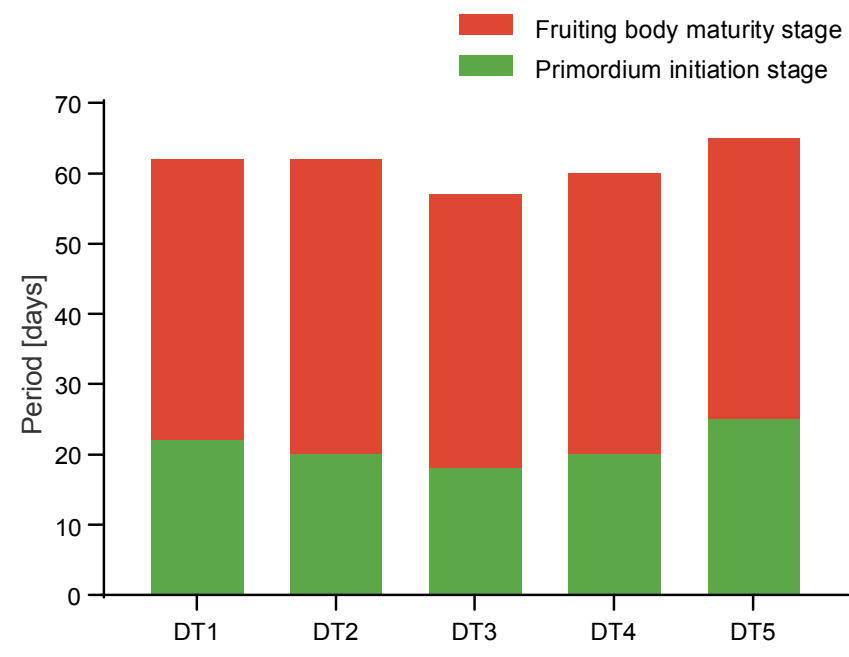

Fig. 3. Growth period of Cordyceps militaris strains in artificial cultivation condition

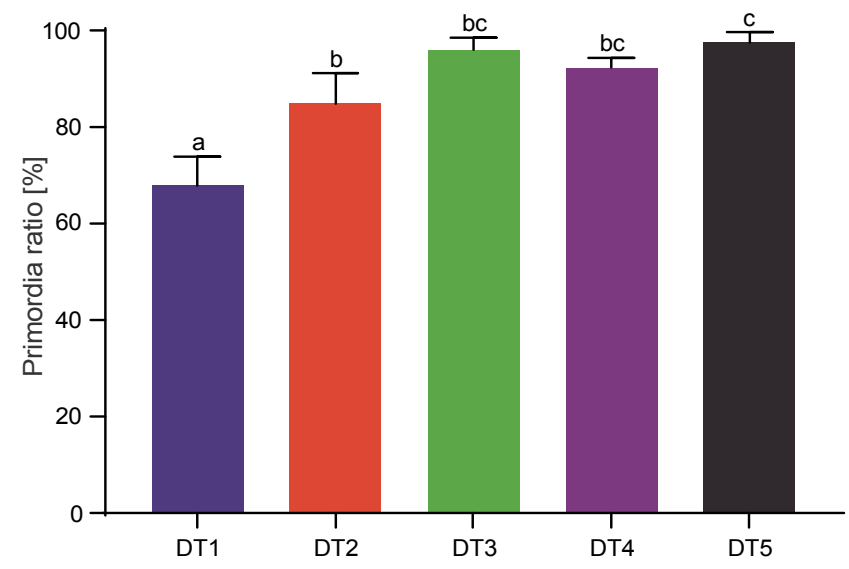

Fig. 4. Primordia ratio of Cordyceps militaris strains in artificial cultivation condition; bars with different letters show significant differences at $P<0.05$

\section{Discussion}

Nutrients are well known to be the main factor that noticeably affect the growth of mushrooms (Nguyen et al., 2019). To meet all the nutrient requirements for the vegetative growth of $C$. militaris, various types of media were developed for its isolation, identification, and preservation (Shrestha et al., 2006). For the vegetative growth of $C$. militaris, sucrose, beef extract, zinc chloride, and folic acid were found to be optimal carbon, nitrogen, mineral, and vitamin sources, respectively (Pathania et al., 2015). As highlighted by Shrestha et al. (2006), SDAY, SMAY (maltose $40 \mathrm{~g} / 1$, peptone $10 \mathrm{~g} / \mathrm{l}$, yeast extract $10 \mathrm{~g} / \mathrm{l}$, agar $15 \mathrm{~g} / \mathrm{l}$ ), and CZYA (sucrose $30 \mathrm{~g} / \mathrm{l}$, yeast extract $5 \mathrm{~g} / \mathrm{l}, \mathrm{NaNO}_{3} 3 \mathrm{~g} / \mathrm{l}, \mathrm{MgSO}_{4} \cdot 7 \mathrm{H}_{2} \mathrm{O}$ $0.5 \mathrm{~g} / \mathrm{l}, \mathrm{KCl} 0.5 \mathrm{~g} / \mathrm{l}, \mathrm{KH}_{2} \mathrm{PO}_{4} 0.01 \mathrm{~g} / 1, \mathrm{~K}_{2} \mathrm{HPO}_{4} 1 \mathrm{~g} / \mathrm{l}$, and agar $20 \mathrm{~g} / \mathrm{l}$ ) were the optimal media for the vegetative growth of $C$. militaris. Therefore, SDAY was used as the medium to investigate the growth characteristics of C. militaris in the present study. As described previously by Shrestha et al. (2006) regarding mycelial texture, C. militaris showed cottony-type mycelial texture from the initial stage in the dark condition. The mycelial morphology of $C$. militaris growth on the solid-state medium was identified as wooly in texture, creamish white in color at the initial stage, which later changed to light brown at the mature stage (Pathania et al., 2015). In the present study, the five investigated strains showed floccose in terms of texture. Because of different culture periods, genotypes (Nopparat et al., 2018), and growth medium, the characteristics of the mycelium were considerably different for each of the $C$. militaris strains.

Unlike solid culture, submerged cultivation of fungi has several distinct advantages such as time-saving, enhanced bioactive compound content, and efficient biomass production. Therefore, submerged cultivation is becoming increasingly popular as a useful method for artificial field production (Jin et al., 2017). However, many studies emphasized that not all species of the higher fungi are capable of growing well as mycelial cultures in bioreactors (Turlo, 2014). During the submerged cultivation, the growth forms of the mycelium are directly linked to several factors such as culture medium, initial $\mathrm{pH}$, inoculation size, aeration rate, and agitation speed. Generally, under fermentation conditions, filamentous and/or pelleted forms could be observed as growth forms (Park et al., 2002). In the present study, all the five strains were able to grow and form both filamentous and pelleted forms in submerged culture conditions. Therefore, it was concluded that submerged cultivation could be used as a spawn production technology for $C$. militaris cultivation at the industrial scale. As reported by Kim et al. (2003), during submerged cultivation of $C$. militaris, the cells formed mainly pellets at the initial stage and then maintained this growth form almost constant during the entire fermentation period. Several parameters such as diameter, circularity, roughness, and compactness are normally estimated to characterize pellet morphology (Park et al., 2002). Among these characteristics, pellet size is found to be one of the most important parameters. As reported previously, the pellet size greatly affects the transportation of oxygen and nutrients into the core region of pel- 

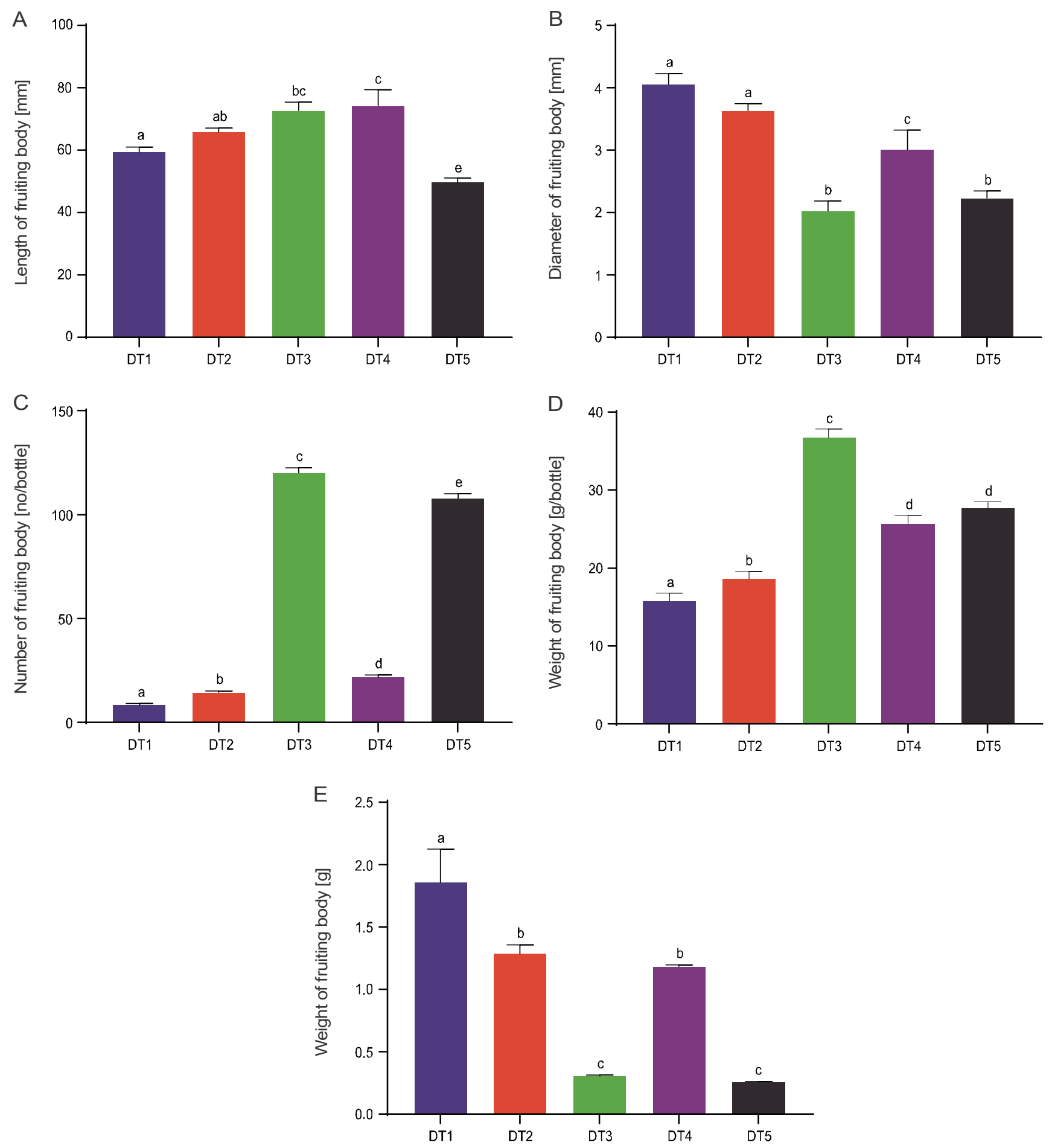

Fig. 5. Characteristics of the fruiting body of Cordyceps militaris strains; bars with different letters show significant differences at $P<0.05$

let cells. The lack of oxygen and nutrients in the center leads to the death of large pellet cells. Thus, pellets with a small size are advantageous (Turlo, 2014). The size of pellet cells during incubation is strictly related to several factors such as agitation course, inoculum size, and carbon concentration in the medium (Turlo, 2014).
For the artificial cultivation of $C$. militaris, cottonseed shells, cob particles (Long, 2016), brown rice, German millet, and millet (Kim et al., 2010) could be used as the basal substrate and nutritional sources. Compared to other substrates, brown rice, German millet, and millet exhibitedhigher stromata lengths of approximately $50 \mathrm{~mm}$ 
A
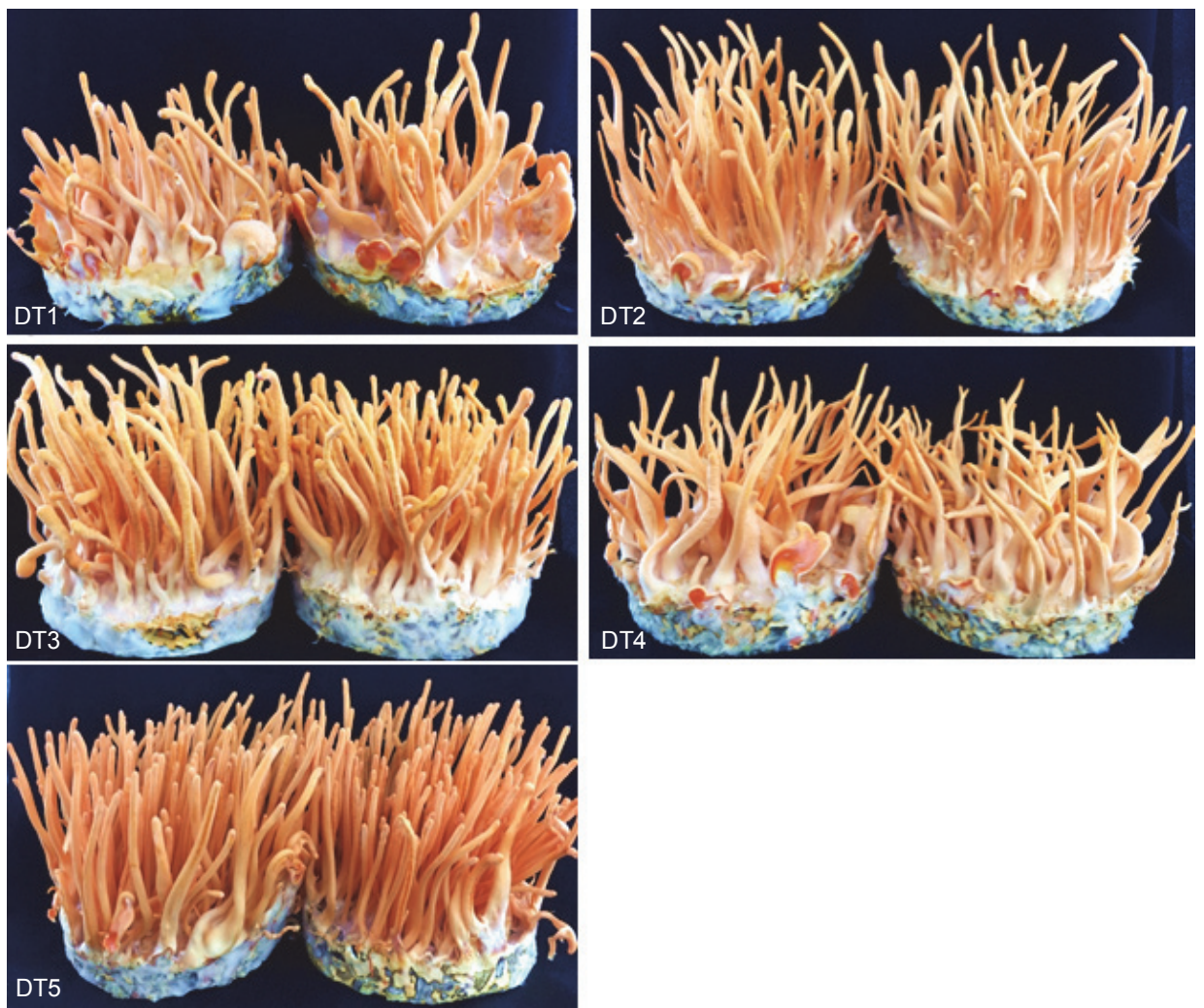

B

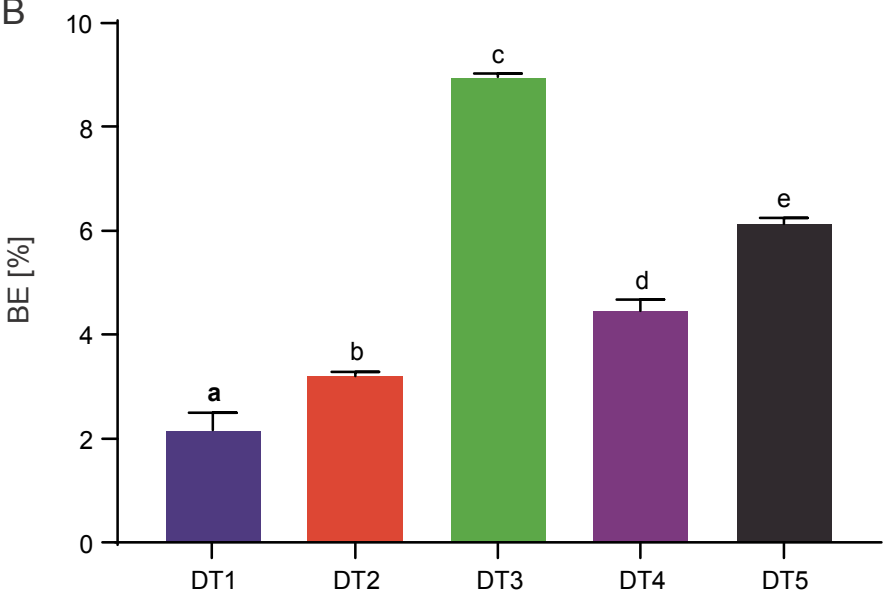

Fig. 6. A) Fruiting bodies and B) biological efficiency of $C o r$ dyceps militaris strains; bars with different letters show significant differences at $P<0.05$

(Kim et al., 2010). Brown rice showed the highest yield of fruiting bodies with 6-7 $\mathrm{g}$ of fresh weight per bottle (Kim et al., 2010). Thus, brown rice supplemented with silkworm pupae powder as the main substrate was used in the present study. All five strains investigated in this study showed the ability to form and develop primordia to the maturity stage.

According to Lee et al. (2015), the fruiting bodies of strain Dowonhongcho and strain Yedang 3 were formed on 15 days after inoculation. Under favorable conditions, the period for substrate colonization, primordia formation, and harvest of $C$. militaris were 5-6 days, 12 days, and 45-50 days after inoculation, respectively (Du et al., 2010). Among all strains used for this study, the earliest primordia formation was recorded for strain DT3 with 18 days. This may be due to the different genotype and culture conditions. The length of the fruiting body is one of the most important morphological characteristics that affect the commercial value of $C$. militaris. As reported by Kim et al. (2010), higher lengths with fewer fruiting 
A

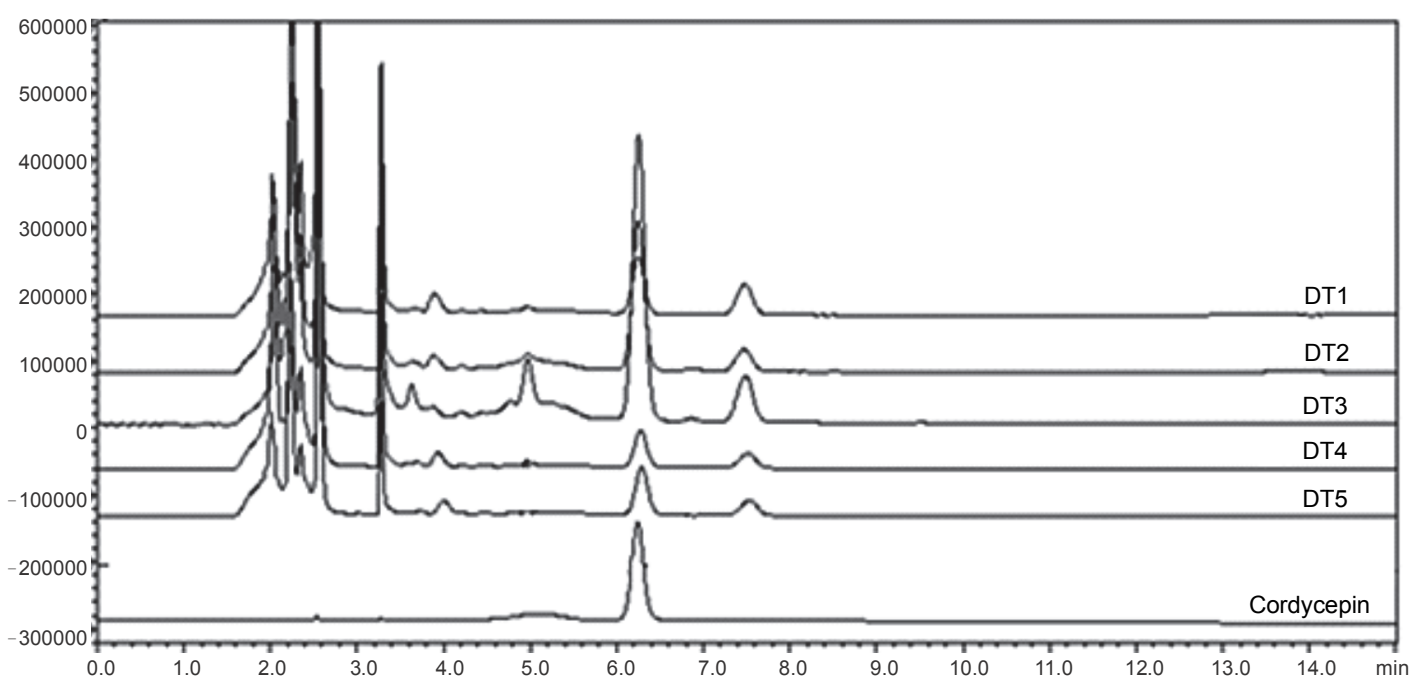

B

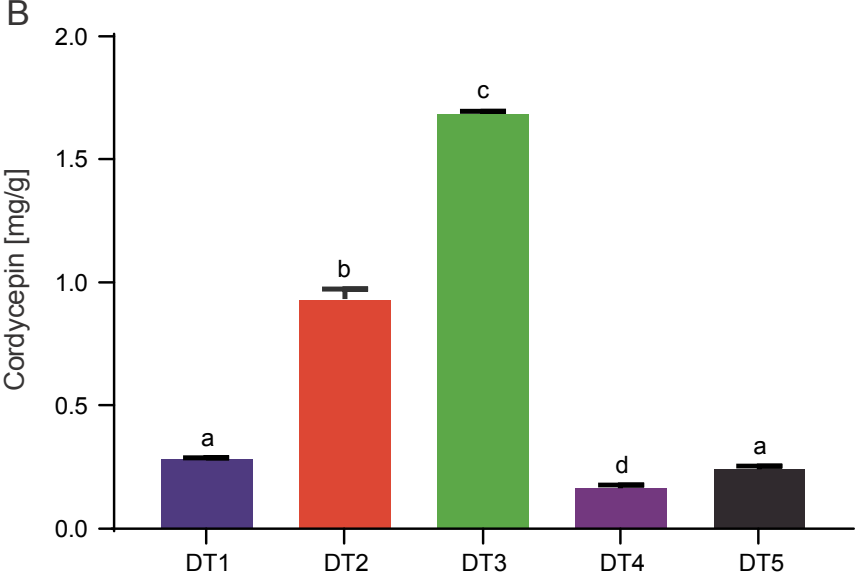

Fig. 7. A) HPLC chromatogram of the standard and strains DT1, DT2, DT3, DT4, and DT5, B) comparison of cordycepin content of Cordyceps militaris strains; bars with different letters show significant differences at $P<0.05$

bodies often lead to reduced biomass and thus affect commercial value. However, higher biomass of fruiting bodies is related to the appearance of abnormally shaped fruiting bodies, thereby resulting in decline in commercial value (Kim et al., 2010). The size of the fruiting body ranged from 4.2 to $7.8 \mathrm{~cm}$ (Pathania et al., 2015). In our present study, strains DT4 and DT3 exhibited the highest length of the fruiting body with $74.23 \pm 5.13 \mathrm{~mm}$ and $72.63 \pm 2.62 \mathrm{~mm}$, respectively.

Cordycepin, a well-known biomarker of genus Cordyceps (Ghatnur et al., 2015; Guo et al. 2016), has been widely used as a bioactive compound in immunological, hepatic, renal, and cardiovascular disorders and as an anticancer agent (Jin et al., 2018). The content of cordycepin in C. militaris is related to several factors such as culture condition, growth stage, and ingredients of medium. Thus, to enhance the production yield of cordy- cepin in $C$. militaris culture, numerous studies have been conducted to optimize culture conditions. Through optimal culture conditions (10 g/l glucose, $10 \mathrm{~g} / 1$ peptone, $1.0 \mathrm{~g} / 1 \mathrm{MgSO}_{4} \cdot 7 \mathrm{H}_{2} \mathrm{O}, 1.0 \mathrm{~g} / 1 \mathrm{~K}_{2} \mathrm{HPO}_{4}$, and $1.0 \mathrm{mg} / 1$ $\alpha$-naphthylacetic acid), the average content of cordycepin isolated from fruiting bodies reached $9.17 \pm 0.09 \mathrm{mg} / \mathrm{g}$ (Wen et al., 2014). C. militaris strains cultivated in silkworm exhibited the highest cordycepin concentration $(4.17 \pm 1.66 \mathrm{mg} / \mathrm{g})$ followed by brown rice medium $(2.98$ $\pm 1.41 \mathrm{mg} / \mathrm{g}$ ), while $C$. militaris cultivated in PDB showed the least content of cordycepin with 1.08 $\pm 0.73 \mathrm{mg} / \mathrm{g}$ (Kang et al., 2017). This may be because the silkworm pupae medium contains a high concentration of proteins and low carbohydrate content (Lee et al., 2017; Guo et al., 2016). Strain KSP8 with a high content of cordycepin $(6.63 \mathrm{mg} / \mathrm{g})$ was developed by mating (Kang et al., 2017). C. militaris cultivated on different 
media could produce cordycepin in amounts ranging from 1 to $14 \mathrm{~g}$ (Cho et al., 2010). The contents of cordycepin, niacin, and potassium isolated from the fruiting bodies of $C$. militaris cultivated on soy power media were higher than those obtained from the strain cultivated on other media (Cho et al., 2010). The cordycepin content of strain Haizhou 1 was $24.98 \mathrm{mg} / \mathrm{g}$ DW (Du et al., 2010). In the present study, the cordycepin content of the five strains cultivated in rice medium ranged from $0.24 \mathrm{mg} / \mathrm{g}$ (DT5) to $1.68 \mathrm{mg} / \mathrm{g}$ (DT3). Therefore, further studies are needed to establish optimal culture conditions for cordycepin production.

\section{Conclusions}

All the tested fungal strains in the present study exhibited the ability to grow mycelium on both SDAY (dextrose $20 \mathrm{~g} / \mathrm{l}$, yeast extract $5 \mathrm{~g} / \mathrm{l}$, peptone $5 \mathrm{~g} / \mathrm{l}$, and agar $15 \mathrm{~g} / \mathrm{l}$ ) and liquid (peptone $1 \mathrm{~g} / \mathrm{l}$, yeast extract $1 \mathrm{~g} / 1$, $\left.\mathrm{MgSO}_{4} \cdot 7 \mathrm{H}_{2} \mathrm{O} 1 \mathrm{~g} / 1, \mathrm{KH}_{2} \mathrm{PO}_{4} 2 \mathrm{~g} / \mathrm{l}\right)$ media. Compared with other strains, DT3 exhibited the highest biological growth efficiency $(8.95 \pm 0.07 \%)$ and cordycepin content $(1.68 \mathrm{mg} / \mathrm{g})$. Therefore, strain DT3 could be considered as a potential strain for commercial cultivation.

\section{Acknowledgments}

This study was partially funded by Vietnam National University of Agriculture as a "research working group project".

\section{References}

Akata I., Kabaktepe Ş., Akgül H. (2016) Cordyceps militaris the first record from family cordycipitaceae in Turkey. Kastamonu Uni., Orman Fakültesi Dergisi. 16(1): 280-284.

Chamyuang S., Owatworakit A., Honda Y. (2019) New insights into cordycepin production in Cordyceps militaris and applications. Ann. Transl. Med. 7(3): 9-11.

Chen Y.S., Liu B.L., Chang Y.N. (2011) Effects of light and heavy metals on Cordyceps militaris fruit body growth in rice grain-based cultivation. Korean J. Chem. Eng. 28(3): $875-879$

Chiang S., Liang Z., Wang Y., Liang C. (2017) Effect of lightemitting diodes on the production of cordycepin, mannitol and adenosine in solid-state fermented rice by Cordyceps militaris. J. Food Compos. Anal. 60: 51-56.

Chiu C.-P., Hwang T.-L., Chan Y., El-Shazly M., Wu T.-Y., Lo I.W., Hsu Y.-M., Lai K.-H., Hou M.-F., Yuan S.-S., Chang F.R., Wu Y.-C. (2016) Research and development of Cordyceps in Taiwan. Food Sci. Hum. Wellness 5(4): 177-185.

Cho S.M., Park H.J., Seo G.-S., Hong J.-D. (2010) Effect of medias composition on the Cordycepin and content Nutri- tional Components of Cordyceps militaris. Korean J. Mycol. 37(2): 161-166.

Cui J.D. (2015) Biotechnological production and applications of Cordyceps militaris, a valued traditional Chinese medicine. Crit. Rev. Biotechnol. 35(4): 475-484.

Das S.K., Masuda M., Sakurai A., Sakakibara M. (2010) Medicinal uses of the mushroom Cordyceps militaris: current state and prospects. Fitoterapia 81(8): 961-968.

Du A.L., Zhang X., Zhang H.Z. (2010) A new high cordycepin Cordyceps militaris cultivar 'Haizhou 1'. Acta Hortic. Sin. 37: 1373-1374.

Ghatnur S., Parvatam G., Balaraman M. (2015) Culture conditions for production of biomass, adenosine, and cordycepin from Cordyceps sinensis CS1197: Optimization by desirability function method. Pharmacogn. Mag. 11(44): 448.

Guo M., Guo S., Huaijun Y., Bu N, Dong C.-H. (2016) Comparison of major bioactive compounds of the caterpillar medicinal mushroom, Cordyceps militaris (Ascomycetes), fruiting bodies cultured on wheat substrate and pupae. Int. J. Med. Mushrooms. 18(4): 327-336.

Hong I., Kang P., Kim K. et al (2010) Fruit body formation on silkworm by Cordyceps militaris. Mycobiology 38(2): 128-132.

Jin J., Zhong C., Qin Y., Cai Y., Zhen L. et al. (2017) A new cordycepin-producing caterpillar fungus Ophiocordyceps xuefengensis with artificial infection to the host, cultivation of mycelia and stromata. FEMS Microbiol. Lett. 364(20): 1-8.

Jin Y., Meng X., Qiu Z., Su Y., Yu P., Qu P. (2018) Anti-tumor and anti-metastatic roles of cordycepin, one bioactive compound of Cordyceps militaris. Saudi J. Biol. Sci. 25(5): 991-995.

Kang N., Lee H.H., Park I., Seo Y.S. (2017) Development of high cordycepin-producing cordyceps militaris strains. Mycobiology 45(1): 31-38.

Kim S., Shrestha B., Sung G., Han S., Sung J. (2010) Optimum conditions for artificial fruiting body formation of Cordyceps cardinalis. Mycobiology 38(2): 133-136.

Kim S.W., Xu C.P., Hwang H.J., Choi J.W., Kim C.W., Yun J.W. (2003) Production and characterization of exopolysaccharides from an enthomopathogenic fungus Cordyceps militaris NG3. Biotechnol. Prog. 19(2): 428-435.

Lee B., Lee M.-A., Kim Y.-G., Lee K., Choi Y., Lee B. (2015) Varietal characteristics of cross-bred Cordyceps militaris “Dowonhongcho". J. Mushrooms. 13(3): 151-156.

Lee B., Lee M., Kim Y., Lee S., Choi Y., Lee B. (2010) Varietal characteristics of new Cordyceps militaris 'Dowonhongcho 2ho' improved by mating type molecular markers. J. Mushrooms15(3): 111-117.

Lee H.H., Kang N., Park I., Park J., Kim I. et al. (2017) Characterization of newly bred Cordyceps militaris strains for higher production of cordycepin through HPLC and URPPCR analysis. J. Microbiol. Biotechnol. 27(7): 1223-1232.

Lin Q., Long L., Wu L., Zhang F., Wu S. (2017) Evaluation of different agricultural wastes for the production of fruiting 
bodies and bioactive compounds by medicinal mushroom. J. Sci. Food Agric. 97(10): 3476-3480.

Ngo N.X., Nguyen B.T.T., Le V.V., Nguyen L.T., Nguyen T.T., Nguyen Q.D. (2019) Morphological characteristics, yield performance, and medicinal value of some Lingzhi mushroom (Ganoderma lucidum) strains cultivated in Tam Dao, Vietnam. Vietnam J. Agric. Sci. 2(1): 321-331.

Nguyen B.T.T., Ngo N.X., Le V.V., Nguyen L.T., Kana R., Nguyen H.D. (2019) Optimal culture conditions for mycelial growth and fruiting body formation of Ling Zhi mushroom Ganoderma lucidum strain GA3. Vietnam J. Sci. Technol. 61(1): 62-67.

Nopparat J., Sujipuli K., Chatdumrong W. (2018) Morphological and molecular analysis of commercial Cordyceps militaris strains in Thailand. Int. J. Biosci. 6655: 378-386.

Park J.P., Kim S.W., Hwang H.J., Yun J.W. (2001) Optimization of submerged culture conditions for the mycelial growth and exo-biopolymer production by Cordyceps militaris. Lett. Appl. Microbiol. 33(1): 76-81.

Park J.P., Kim Y.M., Kim S.W., Hwang J., Cho Y.J. et al. (2002) Effect of aeration rate on the mycelial morphology and exo -biopolymer production in Cordyceps militaris. Process. Biochem. 37(11): 1257-1262.

Pathania P., Joshi M., Sagar A. (2015) Morphological, physiological and molecular studies on wildly collected Cordyceps militaris from North West Himalayas, India. Eur. J. Biotechnol. Biosci. 3(1): 53-62.

Shrestha B., Han S.K., Sung J.M., Sung G.H. (2012a) Fruiting body formation of Cordyceps militaris from multi-ascospore isolates and their single ascospore progeny strains. Mycobiology 40(2): 100-106.

Shrestha B., Lee W.-H., Han S.-K., Sung J.-M. (2006) Observations on some of the mycelial growth and pigmentation characteristics of Cordyceps militaris isolates. Mycobiology 34(2): 83-91.

Shrestha B., Zhang W., Zhang Y. (2012b) The medicinal fungus Cordyceps militaris: research and development. The medicinal fungus Cordyceps militaris: research and development. Mycol. Progress. 11(3): 599-614.

Sun H., Hu T., Guo Y., Liang Y. (2018) Preservation affects the vegetative growth and fruiting body production of Cordyceps militaris. World J. Microbiol. Biotechnol. 34(11): 166.

Turlo J. (2014) The biotechnology of higher fungi - current state and perspectives. Folia Biol. Oecol. 10: 49-65.

Wang F., Song X., Dong X., Zhang J., Dong C. (2017) DASHtype cryptochromes regulate fruiting body development and secondary metabolism differently than $\mathrm{CmWC}-1$ in the fungus Cordyceps militaris. Appl. Microbiol. Biotechnol. 101: 4645-4657.

Wen T.C., Li G.R., Kang J.C., Kang C., Hyde K.D. (2014) Optimization of solid-state fermentation for fruiting body growth and cordycepin production by Cordyceps militaris. Chiang Mai J. Sci. 41(4): 858-872.

Yang S., Jin L., Ren X., Lu J., Meng Q. (2014) Optimization of fermentation process of Cordyceps militaris and antitumor activities of polysaccharides in vitro. J. Food Drug Anal. 22(4): 468-476.

Yang T., Guo M., Yang H., Guo S., Dong C. (2016) The bluelight receptor $\mathrm{Cm} W \mathrm{C}-1$ mediates fruit body development and secondary metabolism in Cordyceps militaris. Appl. Microbiol. 100(2): 743-755.

Yin J., Xin X., Weng Y., Gui Z. (2017) Transcriptome-wide analysis reveals the progress of Cordyceps militaris subculture degeneration. Plos One. 12(10): 1-14.

Zhang J., Wen C., Duan Y., Zhang H., Ma H. (2019) Advance in Cordyceps militaris (Linn) Link polysaccharides: isolation, structure, and bioactivities: a review. Int. J. Biol. Macromol. 132: 906-914.

Zhao J., Xie J., Wang L.Y., Li S.P. (2014) Advanced development in chemical analysis of Cordyceps. J. Pharm. Biomed. Anal. 87: 271-289. 